\title{
Notes on the Cevian nest property and the Newton property of projective planes
}

\author{
Zoltán Szilasi \\ Zoltán Szilasi received his Ph.D. from the University of Debrecen, Hungary, in 2010 \\ Currently he is a temporary lecturer at the University of Debrecen. His research inter- \\ ests are classical and modern projective geometry and related topics.
}

\section{Preliminaries}

First of all, we recall some basic definitions and constructions. By an incidence geometry we mean a triple $(\mathbf{P}, \mathbf{L}, I)$ consisting of a set $\mathbf{P}$ of points, a set $\mathbf{L}$ of lines and a relation $I \subset \mathbf{P} \times \mathbf{L}$ called incidence. Two incidence geometries $\left(\mathbf{P}_{\mathbf{1}}, \mathbf{L}_{\mathbf{1}}, I_{1}\right)$ and $\left(\mathbf{P}_{\mathbf{2}}, \mathbf{L}_{\mathbf{2}}, I_{2}\right)$ are said to be isomorphic, if there are bijections $\varphi_{1}: \mathbf{P}_{\mathbf{1}} \rightarrow \mathbf{P}_{\mathbf{2}}$ and $\varphi_{2}: \mathbf{L}_{\mathbf{1}} \rightarrow \mathbf{L}_{\mathbf{2}}$ such that $(A, l) \in I_{1}$ if and only if $\left(\varphi_{1}(A), \varphi_{2}(l)\right) \in I_{2}$. Such a pair of bijections is called an isomorphism from $\left(\mathbf{P}_{\mathbf{1}}, \mathbf{L}_{\mathbf{1}}, I_{1}\right)$ onto $\left(\mathbf{P}_{\mathbf{2}}, \mathbf{L}_{\mathbf{2}}, I_{2}\right)$. A collineation is an isomorphism from an incidence geometry onto itself. If $(A, l)$ is a point-line pair and $(A, l) \in I$, we say that " $A$ is incident with $l$ ", " $A$ lies on $l$ ", " $l$ passes through $A$ ", and so on; and we write $A I l$ or II $A$. However, all incidence geometries are isomorphic to an incidence geometry whose lines are sets of points, so instead of $(A, l) \in I$ we may also write (and think) $A \in l$. Points that are incident with a common line are called collinear, lines that pass through the same point are called concurrent. Throughout the paper the word "distinct" will be understood

Unter den klassischen Inzidenzsätzen der ebenen projektiven Geometrie gehören die Sätze von Pappos und Desargues sicherlich zu den prominentesten Vertretern. Im Rahmen der Einführung abstrakter ebener Inzidenzgeometrien kann man sich nun fragen, welche Folgerungen aus der Gültigkeit der Sätze von Pappos und/oder Desargues für die zugrunde gelegte Inzidenzgeometrie gezogen werden können. So wird in der vorliegenden Arbeit beispielsweise das folgende Ergebnis unter der Voraussetzung der Gültigkeit des Satzes von Pappos bewiesen: Ist $A^{\prime} B^{\prime} C^{\prime}$ bzw. $A^{\prime \prime} B^{\prime \prime} C^{\prime \prime}$ ein in das Dreieck $A B C$ bzw. $A^{\prime} B^{\prime} C^{\prime}$ einbeschriebenes Dreieck, so dass die Dreiecke $A B C$ und $A^{\prime} B^{\prime} C^{\prime}$ bzw. $A^{\prime} B^{\prime} C^{\prime}$ und $A^{\prime \prime} B^{\prime \prime} C^{\prime \prime}$ zueinander perspektivisch sind, so sind es auch die Dreiecke $A B C$ und $A^{\prime \prime} B^{\prime \prime} C^{\prime \prime}$ 
whenever a special number is mentioned. On the other hand, a phrase like "let $A$ and $B$ be points" includes the possibility $A=B$.

An incidence geometry is a projective plane if

(P1) for every pair of distinct points $A$ and $B$ there is a unique line incident with $A$ and $B$ (we denote this line by $\overleftrightarrow{A B}$ );

(P2) for every pair of distinct lines $m$ and $n$ there is a unique point incident with $m$ and $n$ (we denote this point by $m \cap n$ );

(P3) there are four points no three of which are collinear.

In a projective plane an ordered triple of noncollinear points is a triangle. Then the points are called the vertices, and the lines joining the three possible distinct pairs of vertices are called sides. We say that two triangles $A B C$ and $A^{\prime} B^{\prime} C^{\prime}$ are centrally perspective from a point $O$ if the lines $\overleftrightarrow{A A^{\prime}}, \overleftrightarrow{B B^{\prime}}$, and $\overleftrightarrow{C C^{\prime}}$ are incident with $O$. The triangles are called axially perspective from a line $l$ if the points $\overleftrightarrow{A B} \cap \overleftrightarrow{A^{\prime} B^{\prime}}, \overleftrightarrow{A C} \cap \overleftrightarrow{A^{\prime} C^{\prime}}$, and $\overleftrightarrow{B C} \cap \overleftrightarrow{B^{\prime} C^{\prime}}$ are incident with $l$. An ordered quadruple $A B C D$ of points is called a four-point, if no three of the points are collinear. A complete quadrangle is a four-point $A B C D$, together with the six lines, called sides, determined by pairs of the four points. The points $\overleftrightarrow{A B} \cap \overleftrightarrow{C D}$, $\overleftrightarrow{A C} \cap \overleftrightarrow{B D}, \overleftrightarrow{A D} \cap \overleftrightarrow{B C}$ are the diagonal points of the quadrangle.

The following incidence properties are fundamental in the theory of projective planes:

(P) If $A, B, C$ and $A^{\prime}, B^{\prime}, C^{\prime}$ are triples of distinct points on distinct lines $l$ and $l^{\prime}$, respectively, and $l \cap l^{\prime}$ is different from all six points, then the points $\overleftrightarrow{A B^{\prime}} \cap \overleftrightarrow{A^{\prime} B}$, $\overleftrightarrow{A C^{\prime}} \cap \overleftrightarrow{A^{\prime} C}$, and $\overleftrightarrow{B C^{\prime}} \cap \overleftrightarrow{B^{\prime} C}$ are collinear.

(D) If two triangles are perspective from a point, then they are perspective from a line.

(F) There is no complete quadrangle whose diagonal points are collinear.

Projective planes that satisfy (P) or (D) are said to be Pappian and Desarguesian, respectively. If in a projective plane (F) is true, we say that it has the Fano property. By a famous theorem of Hessenberg every Pappian projective plane is Desarguesian. (For a neat proof of this fact we refer to [7], other complete proofs can be found in [3], [8], [9], or [11].)

Let $\mathbb{K}$ be a skewfield. Let $\left(x_{1}, x_{2}, x_{3}\right)$ and $\left(y_{1}, y_{2}, y_{3}\right)$ be two triples of elements of $\mathbb{K}$ different from $(0,0,0)$. We call them equivalent, if there is a $\lambda \in \mathbb{K}$ such that $x_{i}=\lambda y_{i}$ for all $i \in\{1,2,3\}$. Let the elements of $\mathbf{P}$ and the elements of $\mathbf{L}$ be the equivalence classes induced by this relation. We denote the equivalence class represented by $\left(x_{1}, x_{2}, x_{3}\right)$ by $\left[x_{1}, x_{2}, x_{3}\right]$. We say that $\left(\left[x_{1}, x_{2}, x_{3}\right],\left[e_{1}, e_{2}, e_{3}\right]\right) \in I$ if $x_{1} e_{1}+x_{2} e_{2}+x_{3} e_{3}=0$. Then $(\mathbf{P}, \mathbf{L}, I)$ is a projective plane, this is the projective plane over $\mathbb{K}$. Equivalently, the points of this projective plane are the one-dimensional subspaces of the three-dimensional vector space $\mathbb{K}^{3}$ over $\mathbb{K}$, the lines are the two-dimensional subspaces of the same vector space, and the incidence is the subset relation. If a projective plane is isomorphic to the projective plane over $\mathbb{K}$ we say that it can be coordinatized by the skewfield $\mathbb{K}$.

It can be shown (see e.g. [3], [6], [9]) that a projective plane is Desarguesian if and only if it can be coordinatized by a skewfield; a projective plane is Pappian if and only if it can be 
coordinatized by a field; and a Desarguesian projective plane satisfies the Fano property if and only if it can be coordinatized by a skewfield in which $1+1 \neq 0$.

In a Desarguesian projective plane we can choose a basis of the corresponding vector space such that the vertices of an arbitrarily chosen complete quadrangle $A B C D$ are coordinatized by $[1,0,0],[0,1,0],[0,0,1]$, and $[1,1,1]$, respectively.

In a projective plane we call a quadruple $(A B C D)$ of collinear points a harmonic tetrad, if there is a complete quadrangle such that $A$ and $C$ are vertices, $D$ is a diagonal point of the complete quadrangle, and the line of the other two diagonal points intersects the line $\overleftrightarrow{A C}$ at $B$. If a Desarguesian projective plane satisfies the Fano property, then for any triples $(A, B, C)$ of distinct collinear points there is a unique point $D$ such that $(A B C D)$ is a harmonic tetrad. This point is called the harmonic conjugate of $C$ with respect to $A$ and $B$.

Let $A\left[a_{1}, a_{2}, a_{3}\right]$ and $B\left[b_{1}, b_{2}, b_{3}\right]$ be two points in the projective plane over a skewfield $\mathbb{K}$. Then any point of $\overleftrightarrow{A B}$ can be represented by a vector of the form $\alpha\left(a_{1}, a_{2}, a_{3}\right)+$ $\beta\left(b_{1}, b_{2}, b_{3}\right) ; \alpha, \beta \in \mathbb{K}$, not both of them are equal to zero. If $C$ is represented by $\alpha\left(a_{1}, a_{2}, a_{3}\right)+\beta\left(b_{1}, b_{2}, b_{3}\right)$ and $D$ is represented by $\mu\left(a_{1}, a_{2}, a_{3}\right)+\delta\left(b_{1}, b_{2}, b_{3}\right)$, then $(A B C D)$ is a harmonic tetrad if and only if $\beta(\alpha)^{-1} \mu(\delta)^{-1}=-1$.

\section{The Cevian nest property}

We say that a triangle $A^{\prime} B^{\prime} C^{\prime}$ is inscribed in the triangle $A B C$ if $A^{\prime} \in \overleftrightarrow{B C}, B^{\prime} \in \overleftrightarrow{A C}$, and $C^{\prime} \in \overleftrightarrow{A B}$. A projective plane satisfies the Cevian nest property, if the following is true:

(CN) Let $A^{\prime} B^{\prime} C^{\prime}$ be an inscribed triangle of the triangle $A B C, A^{\prime \prime} B^{\prime \prime} C^{\prime \prime}$ an inscribed triangle of the triangle $A^{\prime} B^{\prime} C^{\prime}$. If $A B C$ and $A^{\prime} B^{\prime} C^{\prime}$ as well as $A^{\prime} B^{\prime} C^{\prime}$ and $A^{\prime \prime} B^{\prime \prime} C^{\prime \prime}$ are centrally perspective, then $A B C$ and $A^{\prime \prime} B^{\prime \prime} C^{\prime \prime}$ are also centrally perspective.

Fig. 1 illustrates the preceding definition.

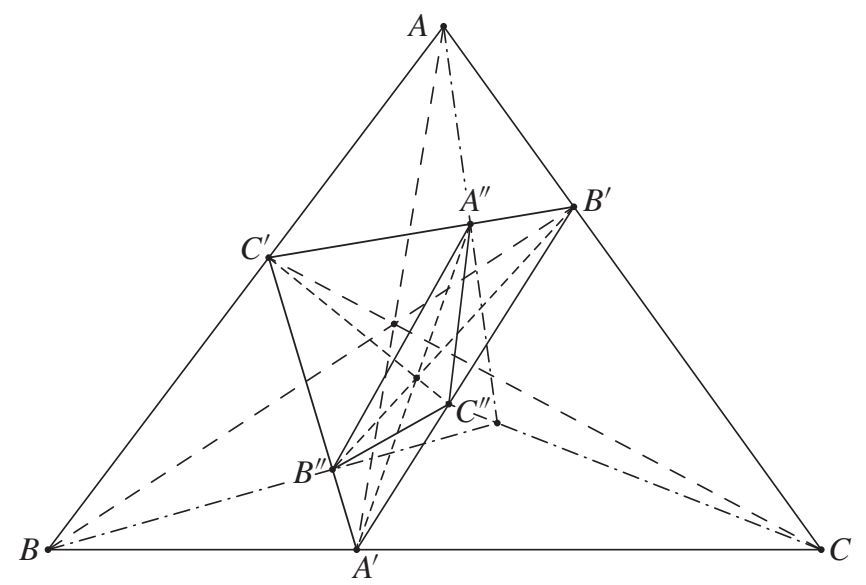

Fig. 1 The Cevian nest property 
It is well-known that $(\mathrm{CN})$ is valid in the classical projective plane (i.e., the projective closure of the Euclidean plane). The first purely incidence geometric proof of this fact was presented by J.-L. Ayme in his manuscript [1]. He proved, in fact, the following:

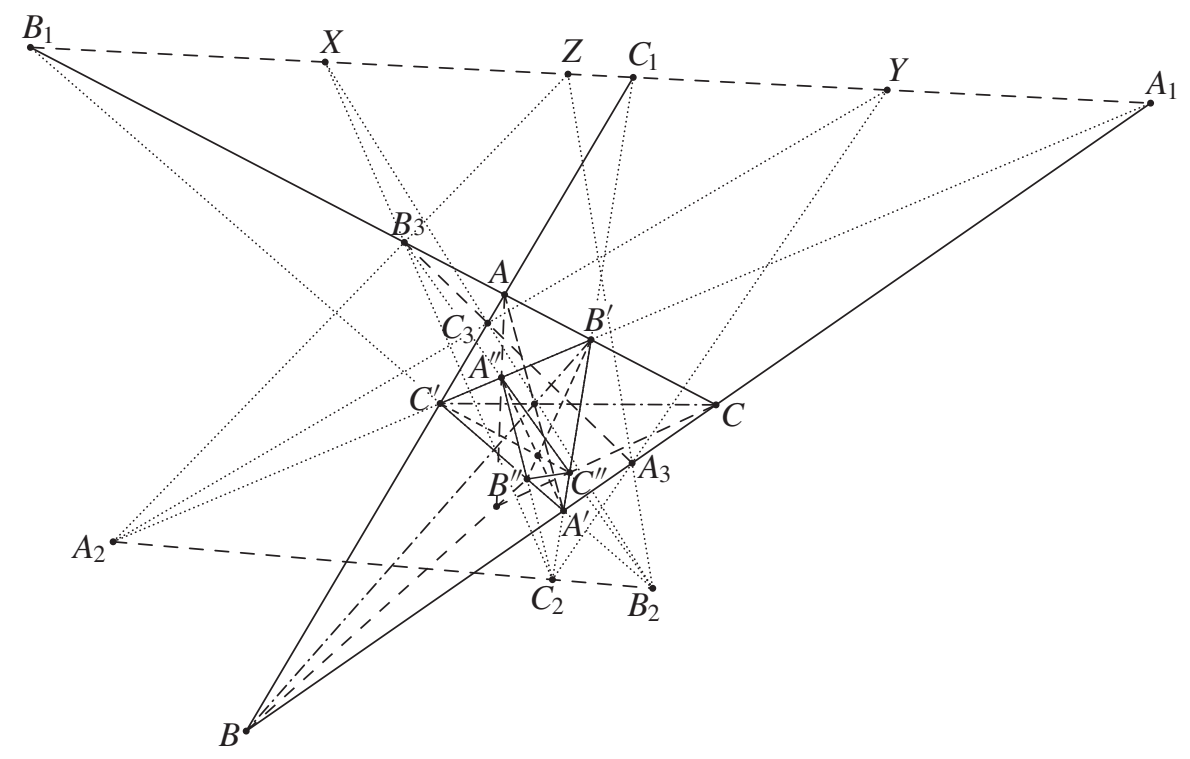

Fig. 2 Proof of Theorem 2.1

Theorem 2.1. If a projective plane is Pappian, then it satisfies the Cevian nest property.

Proof. For the reader's convenience, we reproduce here Ayme's reasoning.

We use that by Hessenberg's theorem the projective plane is Desarguesian as well. Let $A^{\prime} B^{\prime} C^{\prime}$ be an inscribed triangle of $A B C$ and $A^{\prime \prime} B^{\prime \prime} C^{\prime \prime}$ be an inscribed triangle of $A^{\prime} B^{\prime} C^{\prime}$. Suppose that $A B C$ and $A^{\prime} B^{\prime} C^{\prime}, A^{\prime} B^{\prime} C^{\prime}$ and $A^{\prime \prime} B^{\prime \prime} C^{\prime \prime}$ are centrally perspective. (Cf. to Fig. 2.) Let $C_{1}:=\overleftrightarrow{A B} \cap \overleftrightarrow{A^{\prime} B^{\prime}}, B_{1}:=\overleftrightarrow{A C} \cap \overleftrightarrow{A^{\prime} C^{\prime}}$, and $A_{1}:=\overleftrightarrow{B C} \cap \overleftrightarrow{B^{\prime} C^{\prime}}$. By the Desargues property $A_{1}, B_{1}$, and $C_{1}$ are collinear. Similarly, $C_{2}:=\overleftrightarrow{A^{\prime} B^{\prime}} \cap \overleftrightarrow{A^{\prime \prime} B^{\prime \prime}}, B_{2}:=\overleftrightarrow{A^{\prime} C^{\prime}} \cap \overleftrightarrow{A^{\prime \prime} C^{\prime \prime}}$, and $A_{2}:=\overleftrightarrow{B^{\prime} C^{\prime}} \cap \overleftrightarrow{B^{\prime \prime} C^{\prime \prime}}$ are also collinear. We have to show that $C_{3}:=\overleftrightarrow{A B} \cap \overleftrightarrow{A^{\prime \prime} B^{\prime \prime}}$, $B_{3}:=\overleftrightarrow{A C} \cap \overleftrightarrow{A^{\prime \prime} C^{\prime \prime}}$, and $A_{3}:=\overleftrightarrow{B C} \cap \overleftrightarrow{B^{\prime \prime} C^{\prime \prime}}$ are collinear as well.

Since the triangles $A_{1} A_{2} A_{3}$ and $C_{1} C_{3} C_{2}$ are axially perspective from the line incident to $C^{\prime \prime}, B^{\prime \prime}$, and $A^{\prime}$, they are also perspective from a point $Y$. Then $Y$ is incident to the line of the points $A_{1}, B_{1}$, and $C_{1}$. Similarly, the triangles $B_{1} B_{2} B_{3}$ and $A_{1} A_{3} A_{2}$ are perspective from a line, so they are perspective from a point $Z$; finally the triangles $C_{1} C_{2} C_{3}$ and $B_{1} B_{3} B_{2}$ are perspective from a line, therefore they are perspective from a point $X$. Then $Z$ and $X$ are on the line of the points $A_{1}, B_{1}, C_{1}$. Using Pappos property (P) to the collinear triples of points $Z, Y, X$ and $A_{2}, C_{2}, B_{2}$, we find that the points $B^{\prime \prime}, C^{\prime \prime}$, and $A^{\prime \prime}$ are collinear, as was to be proved. 
It is easy to see that in the Desarguesian case $(\mathrm{CN})$ has a meaning only if the Fano property is also satisfied. Indeed, if in a Desarguesian projective plane the Fano property does not hold, then the diagonal points of every complete quadrangle are collinear (for a proof see for example [6]). Therefore, if in such a projective plane $A B C$ is a triangle, $A^{\prime}$ is a point on $\overleftrightarrow{B C}$, and $B^{\prime}$ is a point on $\overleftrightarrow{A C}$, then the diagonals of the complete quadrangle $A B A^{\prime} B^{\prime}$ are collinear, thus the points $C, P:=\overleftrightarrow{A A^{\prime}} \cap \overleftrightarrow{B B^{\prime}}$ and $\overleftrightarrow{A B} \cap \overleftrightarrow{A^{\prime} B^{\prime}}$ are collinear. This means that the point $C^{\prime}:=\overleftrightarrow{C P} \cap \overleftrightarrow{A B}$ coincides with $\overleftrightarrow{A B} \cap \overleftrightarrow{A^{\prime} B^{\prime}}$, so $A^{\prime}, B^{\prime}$, and $C^{\prime}$ are collinear. Consequently, in this case the "triangle" $A^{\prime} B^{\prime} C^{\prime}$ degenerates to a collinear set of points, so $(\mathrm{CN})$ is meaningless.

It is natural to ask whether the usage of the Pappos property in the previous proof is necessary. We answer this question affirmatively.

Theorem 2.2. A Desarguesian projective plane satisfying the Fano axiom has the Cevian nest property if and only if it is Pappian.

Proof. By the previous theorem, every Pappian projective plane satisfies the Cevian nest property. We have only to show that if a Desarguesian projective plane with the Fano property satisfies the Cevian nest property, then it is Pappian. We use the notations of the previous proof. A Desarguesian projective plane can be coordinatized by a skewfield $\mathbb{K}$, and since the Fano property holds, the inequality $1+1 \neq 0$ is true. We will denote the element $1+1$ of $\mathbb{K}$ by 2 . By our assumption it has a multiplicative inverse denoted by $\frac{1}{2}$.

We can choose a basis of $\mathbb{K}^{3}$ such that $A, B$, and $C$ are represented by $(0,1,0),(0,0,1)$, and $(1,0,0)$, respectively, and the center of perspectivity of the triangles $A B C$ and $A^{\prime} B^{\prime} C^{\prime}$ is $P[1,1,1]$. Then, as an easy calculation shows,

$$
A^{\prime}=[1,0,1], \quad B^{\prime}=[1,1,0], \quad C^{\prime}=[0,1,1] .
$$

Let the center of perspectivity of the triangles $A^{\prime} B^{\prime} C^{\prime}$ and $A^{\prime \prime} B^{\prime \prime} C^{\prime \prime}$ be $Q[a, b, 1]$. (We may suppose that $Q$ does not lie on $\overleftrightarrow{A C}$.)

First we calculate a representative vector for the point $A^{\prime \prime}$. The representative vectors of the points incident to $\overleftrightarrow{B^{\prime} C^{\prime}}$ are of form $\alpha_{1}(0,1,1)+\beta_{1}(1,1,0)$. The points of $\overleftrightarrow{A^{\prime \prime} Q}$ can be represented by vectors of the form $\gamma_{1}(1,0,1)+\delta_{1}(a, b, 1)$. So for a representative vector of $A^{\prime}$ we get

$$
\alpha_{1}(0,1,1)+\beta_{1}(1,1,0)=\gamma_{1}(1,0,1)+\delta_{1}(a, b, 1) .
$$

We can choose $\delta_{1}:=1$. Then we have

$$
\left(\beta_{1}, \alpha_{1}+\beta_{1}, \alpha_{1}\right)=\left(\gamma_{1}+a, b, \gamma_{1}+1\right),
$$

which leads to the system of equations

$$
\left.\begin{array}{rl}
\beta_{1} & =\gamma_{1}+a \\
\alpha_{1}+\beta_{1} & =b \\
\alpha_{1} & =\gamma_{1}+1
\end{array}\right\} .
$$


From this we obtain $\gamma_{1}=\frac{1}{2}(b-a-1)$, hence a representative vector for $A^{\prime \prime}$ is

$$
\frac{1}{2}(b-a-1)(1,0,1)+(a, b, 1)=\frac{1}{2}(a+b-1,2 b, b-a+1),
$$

therefore $A^{\prime \prime}$ is the point $[a+b-1,2 b, b-a+1]$.

By a similar calculation,

$$
B^{\prime \prime}=[a-b+1,-a+b+1,2], \quad C^{\prime \prime}=[2 a, a+b-1, a-b+1] .
$$

Using the same technique, we find that the point $\overleftrightarrow{A A^{\prime \prime}} \cap \overleftrightarrow{B B^{\prime \prime}}$ is

$$
\left[a-b+1,-a+b+1,(a-b+1)(a+b-1)^{-1}(-a+b+1)\right] .
$$

The representative vectors of the points that lie on $\overleftrightarrow{C C^{\prime \prime}}$ are of the form

$$
\alpha_{2}(1,0,0)+\beta_{2}(2 a, a+b-1, a-b+1) .
$$

The point that has just been found lies on this line if and only if there are scalars $\alpha_{2}, \beta_{2}$ in $\mathbb{K}$ such that

$$
\begin{aligned}
& \alpha_{2}(1,0,0)+\beta_{2}(2 a, a+b-1, a-b+1) \\
& \quad=\left(a-b+1,-a+b+1,(a-b+1)(a+b-1)^{-1}(-a+b+1)\right),
\end{aligned}
$$

i.e., if the system of equations

$$
\begin{aligned}
\alpha_{2}+2 \beta_{2} a & =a-b+1 \\
\beta_{2}(a+b-1) & =(-a+b+1) \\
\beta_{2}(a-b+1) & =(a-b+1)(a+b-1)^{-1}(-a+b+1)
\end{aligned}
$$

can be solved. The second equation gives $\beta_{2}=(-a+b+1)(a+b-1)^{-1}$, so the desired $\alpha_{2}, \beta_{2}$ exist if and only if the obtained $\beta_{2}$ satisfies the third equation. From this it follows that a Desarguesian projective plane satisfies the Cevian nest property if and only if for all $a, b \in \mathbb{K}$ we have

$$
(-a+b+1)(a+b-1)^{-1}(a-b+1)=(a-b+1)(a+b-1)^{-1}(b-a+1) .
$$

Let $x:=(a+b-1)^{-1}$. Then our previous condition takes the form

$$
(-a+b+1) x(a-b+1)=(a-b+1) x(b-a+1) .
$$

After multiplication and cancelling the opposite terms, we obtain

$$
x a-x b-a x+b x=x b-x a+a x-b x,
$$

which is equivalent to

$$
x(2 a-2 b)=(2 a-2 b) x .
$$

Denoting $2 a-2 b$ by $y$, we get

$$
x y=y x .
$$

Since for all $x, y \in \mathbb{K}$ there are $a, b \in \mathbb{K}$ such that $(a+b-1)^{-1}=x$ and $2 a-2 b=y$, this means that $\mathbb{K}$ is a field, so the projective plane is Pappian. 


\section{The Newton property}

A quadruple $a b c d$ of lines is called a four-line if no three of the lines are concurrent. The six intersection points of the lines are called vertices. The lines

$$
\overleftrightarrow{(a \cap b)(c \cap d)}, \quad \overleftrightarrow{(a \cap c)(b \cap d)}, \quad \overleftrightarrow{(a \cap d)(b \cap c)}
$$

are the diagonal lines of the four-line. A complete quadrilateral is a four-line, together with its six vertices and three diagonal lines.

In the Euclidean geometry the following theorem, due to Newton, is well-known: the midpoints of the diagonals of any quadrangle are collinear (for various proofs, we refer to [10]). On the classical projective plane any two points $A, B$, their midpoint, and the ideal point of $\overleftrightarrow{A B}$ form a harmonic tetrad. So the statement of Newton's theorem may be formulated in projective terms as follows.

(N) Let $a b c d$ be a complete quadrilateral and $l$ be a line. If $P, Q$, and $R$ are the intersections of $l$ and the diagonals of $a b c d$, then the harmonic conjugates of $P, Q$, and $R$ with respect to the corresponding vertices are collinear.

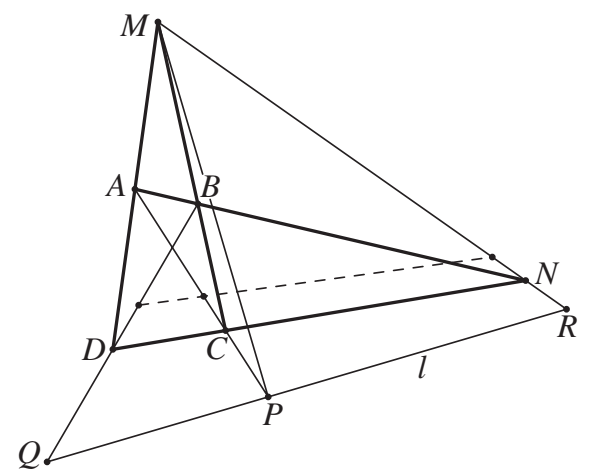

Fig. 3 The Newton property

If $(\mathrm{N})$ is true in a projective plane, we say that it satisfies the Newton property. It is wellknown (see e.g. [5]) that every Pappian projective plane satisfies the Newton property. The classical proof strongly depends on the projective theory of conics, and is a special case of the fact that the poles of a line with respect to the conics touching the lines of a complete quadrilateral are collinear. For Pappian projective planes the Newton property can also be proved using the dual of the theorem of Desargues on complete quadrilaterals. Property $(\mathrm{N})$ has a meaning in every Desarguesian projective plane satisfying Fano's axiom. One may ask whether it is true independently of the Pappos property. Again, the answer is negative.

Theorem 3.1. A Desarguesian projective plane satisfying the Fano axiom has the Newton property if and only if it is Pappian.

Proof. We use the notations of Fig. 3. By our assumptions, the projective plane can be coordinatized by a skewfield $\mathbb{K}$ in which $1+1 \neq 0$ (see above). As in the previous proof, we denote the element $1+1$ of $\mathbb{K}$ by 2 . We can choose a basis of $\mathbb{K}^{3}$ such that four of the 
vertices of the considered quadrilateral are $A[0,0,1], B[1,1,1], C[1,0,0]$, and $D[0,1,0]$. Then the remaining vertices are $M[0,1,1]$ and $N[1,1,0]$.

The points lying on $\overleftrightarrow{A C}$ are of the form $[a, 0,1]$, so we may represent the intersection of $l$ and $\overleftrightarrow{A C}$, i.e., the point $P$, by $(a, 0,1)$. Since $(a, 0,1)=(0,0,1)+a(1,0,0)$, the harmonic conjugate $P^{\prime}$ of $P$ with respect to $A$ and $C$ may be represented by the vector $-(0,0,1)+a(1,0,0)=(a, 0,-1)$, so $P^{\prime}$ is the point

$$
[a, 0,-1] \text {. }
$$

Similarly, the points lying on $\overleftrightarrow{B D}$ have representatives of the form $(1,1,1)+b(0,1,0)$ Let the point $Q$, the intersection of $l$ and $\overleftrightarrow{B D}$, be $[1, b+1,1]$. Then the harmonic conjugate $Q^{\prime}$ of $Q$ with respect to $B$ and $D$ is represented by the vector $-(1,1,1)+b(0,1,0)=$ $(-1, b-1,-1)$, so $Q^{\prime}$ is the point

$$
[-1, b-1,-1] .
$$

We calculate the intersection $R$ of $\overleftrightarrow{M N}$ and $l$. Its representative vectors have the form

$$
\alpha_{1}(0,1,1)+\beta_{1}(1,1,0)=\gamma_{1}(a, 0,1)+\delta_{1}(1, b+1,1) .
$$

We may suppose that $\delta_{1}=1$. Then we obtain the system of equations

$$
\left.\begin{array}{rl}
\beta_{1} & =\gamma_{1} a+1 \\
\alpha_{1}+\beta_{1} & =b+1 \\
\alpha_{1} & =\gamma_{1}+1
\end{array}\right\},
$$

from which $\alpha_{1}=(b-1)(a+1)^{-1}+1, \beta_{1}=(b-1)(a+1)^{-1} a+1$.

$R^{\prime}$, the harmonic conjugate of $R$ with respect to $M$ and $N$, can be represented by a vector of the form $\mu_{2}(0,1,1)+\delta_{2}(1,1,0)$, where $\beta_{1}\left(\alpha_{1}\right)^{-1} \mu_{2}\left(\delta_{2}\right)^{-1}=-1$. Thus a representative vector of $R^{\prime}$ is

$$
\left((b-1)(a+1)^{-1}+1\right)(0,1,1)-\left((b-1)(a+1)^{-1} a+1\right)(1,1,0) .
$$

So $R^{\prime}$ is the point

$$
\left[-(b-1)(a+1)^{-1} a-1,(b-1)(a+1)^{-1}(1-a),(b-1)(a+1)^{-1}+1\right] .
$$

The projective plane satisfies the Newton property if and only if the points $P^{\prime}, Q^{\prime}$, and $R^{\prime}$ are collinear. This is true if and only if there are scalars $\alpha, \beta$ in $\mathbb{K}$ such that

$$
\begin{aligned}
& \alpha(a, 0,1)+\beta(-1, b-1,-1) \\
& \quad=\left(-(b-1)(a+1)^{-1} a-1,(b-1)(a+1)^{-1}(1-a),(b-1)(a+1)^{-1}+1\right)
\end{aligned}
$$

holds. This yields the system of equations

$$
\left.\begin{array}{rl}
\alpha a-\beta & =-(b-1)(a+1)^{-1} a-1 \\
\beta(b-1) & =(b-1)(a+1)^{-1}(1-a) \\
-\alpha-\beta & =(b-1)(a+1)^{-1}+1
\end{array}\right\} .
$$

From the first and the third equation we obtain that $\beta=(1-a)(a+1)^{-1}$. Substituting this into the second equation, we find that the projective plane has the Newton property if 
and only if for all $a, b \in \mathbb{K}$

$$
(1-a)(a+1)^{-1}(b-1)=(b-1)(a+1)^{-1}(1-a) .
$$

Here

$$
\begin{aligned}
(1-a)(a+1)^{-1} & =(-a-1+2)(a+1)^{-1} \\
& =-(a+1)(a+1)^{-1}+2(a+1)^{-1} \\
& =-1+2(a+1)^{-1}
\end{aligned}
$$

and

$$
\begin{aligned}
(a+1)^{-1}(1-a) & =(a+1)^{-1}(-a-1+2) \\
& =(a+1)^{-1}(-(a+1)+2) \\
& =-1+2(a+1)^{-1},
\end{aligned}
$$

taking into account that for any $k \in \mathbb{K}$ we have

$$
2 k=(1+1) k=k+k=k(1+1)=k \cdot 2 .
$$

Denoting $-1+2(a+1)^{-1}$ by $x$, and $b-1$ by $y$, it follows that the Newton property holds if and only if

$$
x y=y x .
$$

Since for all $x, y \in \mathbb{K}$ there are $a, b \in \mathbb{K}$ such that $x=-1+2(a+1)^{-1}$ and $y=b-1$, from this we conclude that $\mathbb{K}$ is a field, so the projective plane is Pappian.

\section{References}

[1] Ayme, J.-L.: The Cevian Nests Theorem. Unpublished manuscript, http: / /pagesperso-orange.fr/jl. ayme, 2008.

[2] Buekenhout, F.: Handbook of Incidence Geometry. North Holland 1995.

[3] Bumcrot, R.J.: Modern Projective Geometry. 1969.

[4] Coxeter, H.S.M.: The Real Projective Plane. Second edition, Cambridge 1955.

[5] Cremona, L.: Elements of Projective Geometry. Third edition, Dover 1960.

[6] Garner, L.E.: An Outline of Projective Geometry. North Holland 1981.

[7] Herzer, A.: Dualitäten mit zwei Geraden aus absoluten Punkten in projektiven Ebenen. Math. Z. 129 (1972), 235-257.

[8] Heyting, A.: Axiomatic Projective Geometry. North-Holland 1980.

[9] Stevenson, F.W.: Projective Planes. W.H. Freeman and Company, 1972.

[10] Tan, K.: Various Proofs of Newton's Theorem. Math. Mag. 39 (1966), 45-58.

[11] Wylie, C.R. jr.: Introduction to Projective Geometry. McGraw-Hill, 1970.

\section{Zoltán Szilasi}

Institute of Mathematics

University of Debrecen

4010 Debrecen, Hungary

e-mail: szilasi.zoltan@science.unideb.hu 